\section{NOTES AND OBSERVATIONS}

or

\section{DISEASES OF THE HEART AND LUNGS.*}

BY T. SHAPTER, M.D., F.R.C.P.,

Senior Physician to the Devon and Exeter Hospi:al, eto.

Having referred to some of the difficulties which may interfere with the recognition of the existence of the normal valvular sounds of the heart, it is now proposed briefly to refer to some of those which, in practice, occasionally embarrass the due appreciation of the presence of murmurs, as also of their precise seat in the heart itself.

In the preceding paper the presence of the normal sounds, and the non-existence of all endocardial murmur, were assumed conditions. In this, on the contrary, the actual presence of one or more of these latter will be assumed, and the circumstances interfering with their due appreciation, most frequently recurring in practice, considered. In doing this, the theory of the origin of these murmurs will be mainly borne in mind.

The practical difficulties that interpose themselves towards ascertaining the presence of a cardiac murmur may be referred chiefly to the following sources; the being overpowered or masked by murmurs emanating from other diseased structures; the being oonfusedly intermingled with other cardiac sounds when there exists a turbulent action in the heart itself; the being thought to be, from other circumstances, not what it really is, but a murmur referable to some other source; the being so slight and so feeble in tone as to be pith the greatest difficulty appreciated as such; or the being entirely ignored from its fleeting and intermitting character.

Of the foreign sounds that interfere with the due appreciation of an existing murmur, the "rales" emanating from a pervading bronchitis are frequent and conspicuous. These are often so similar in their nature, and so pronounced in tone, that, when they coexist, it often becomes a very serious practical difficulty to discriminate, from amidst them, the cardiac murmur. To a certain extent the same may be said of the abnormal respiratory sounds occasionally heard in a pneumonia, and which are found to offer a not infrequent complication with endocardial murmurs, especially when the heart is, coincidently with a pneumonia, the subject of a rheumatic inflammation, and when it especially generates the soft blowing murmur not unusual to the respiratory organs. So similar indeed, at times, are these several murmurs, that perhaps the only reliable mode of distinguishing between them is by procuring, for a short space, an arrest in the breathing. The difficulty here is induced by a quasi similarity of origin; both the respiratory and cardiac murmurs being due to the interference to the even flow or passage of fluid: in the one case, the fluid being the air, in the other, the blood.

Pleuritic and exocardial friction-sounds have been shewn to interfere with the due appreciation of the normal sounds of the heart, and so, in like manner, their presence may render difficult the easy recognition of an existing cardiac murmur by intermingling with, and thus obscuring, it. In these cases the recognition of the cardiac murmur must be sought not only in its retaining under all circumstances its perfect synchronism with the rhythm of the heart, but in

\footnotetext{
* See p. 610 of last volume.
}

the character of the sound itself, and in the presence, may be, of a valvular thrill, independent of the friction fremitus accompanying the exocardial or pleuritic friction-sounds, and in being less circumscribed than are these latter; the cardiac murmur being generally appreciable in the course of the great arteries, as also, when the murmur can be ascertained, in the absence of the true valvular sound. Nevertheless, it is a diagnosis of great difficulty. It is, therefore, not surprising. Dr. Stokes should say (p. 34) that " when it happens, coincidently with the attack of pericarditis, a diseased action is set up in the valves, the determination of the latter may be difficult, during the continuance of the true friction-murmurs. If the valvular sign be, as it commonly is, a bellows-murnur, it may be completely masked by the loudness of the friction-sounds, and only become manifest on their cessation."

A true appreciation of a cardiac murmur may also be interfered with by murmurs originating in the large arteries. Ossific or other deposits, or ulcerations in their coats inducing a roughened state of their surface, or aneurismal dilatations, or any abnormal pressure upon them from surrounding tissues, may each originate murmurs not only loud enough to mask the presence of a valvular murmur, but so similar in rature as to defy a positive differential diagnosis.

A cardiac murmur may be at times fully recognised as a murmur, but may nevertheless be thought not to be cardiac in its origin, but indicative of disease elsewhere, as when it originates a suspicion of exocardial, instead of endocardial, disease, or when it so communicates its sound into the great vessels as to induce the conclusion that the disease has its seat in them. Again, the murmur may be so transmitted through large glandular or cancerous masses, or portions of indurated lung, or even through aneurismal dilatations, as to mislead us as to its true seat and origin.

Difficulty in diagnosis sometimes ensues when there occurs a temporary cessation of the murmur; it may be that there exist the elements for its formation, yet it is only to be heard under certain conditions, and usually those are conditions under which an examination of the heart is not commonly made, namely, after violent or exciting exercise-a period of repose, and there is no murmur to be heard. Sometimes, as disease progresses, the murmur so loses its characteristics as not to be recognised as such ; this is especially the case when the powers of life are failing. Occasionally, also, from double murmurs in each set of valves they become, when the heart is agitated, so mingled and involved as to offer to the ear nothing but a turbulent sound.

Supposing, howerer, that the presence of a cardiac murmur be satisfactorily ascertained, other doubts spring up; such as, to which of the valves is its seat to be referred; then, whether it be caused by physical or by only passing modifications in the heart's structure; or by an alteration in the composition, relative amount, or force in the current, of the blood, irrespective of any alteration in the heart itself.

As regards the ascertaining to which valve a murmur may owe its origin, the first practical difficulty arises from there being a double set of valves in somewhat close contiguity to each other; and this difficulty becomes enhanced, when, from a want of synchronism in the two hearts, a reduplication of murmurs occurs. In the paper preceding this, the subject of the reduplication of the valve-sounds was specially referred to: and it was shown to originate in the fact that there existed a double set of valves, each giving out a sound, in the normal state, so synchronously, as to appear but one sound only, but that if this syn- 
chronism be disturbed, this compound sound is analysed into its elements, and heard as a reduplicated, or cleft, sound. Should this want of synchronism exist when murmurs are present, it is evident that difficulties might be greatly increased; since each set of valves, which, in the former case, is the seat of one sound only, may, in the latter, be the seat not only of one, but of two, murmurs. Hence, while the sounds, usually two, can only be reduplicated into four, the murmurs may be reduplicated, by want of synchronism in the heart's action, into eight. Exception may perhaps be taken to this stated number of eight, as never really occurring, especially as it is affirmed that the reduplication of the sounds is not an accident of advanced disease. Nevertheless, to a certain extent, this is not altogether a theoretical difficulty; for occasionally the same want of synchronism in the systole of the two ventricles, which is rendered patent by the reduplication of the valve-sounds, does occur when murmurs are present, and, if so, tends very much to confuse a clear appreciation of the murmur; but, be this as it may, the fact remains, that there are four valves to be considered, and that, even without this want of synchronism, each of these valves not only may be, but often is the seat of two murmurs, and that towards a correct diagnosis it is necessary not only to ascertain in which valve the murmur originates, but also whether it be with the flow of the blood onward through it, or produced by regurgitation.

In order to ascertain the precise origin of a cardiac murmur, it is necessary first to discriminate which heart is the seat of disease. In this we have not now the practical difficulty that attended these inrestigations a few years since, for experience has taught us that disease, and the accompanying murmurs, belong mainly to the left heart, and that murmurs have rarely their seat in the right heart, or perhaps never, without previous and coexisting existence in the left heart. It may, in fact, be assumed, unless other circumstances indicate the existence of disease in the right heart, that the seat of murmur is in the valves of the left heart.

Sometimes the normal valvular sounds of the right heart may be distinguished from an existing murmur of the left heart. Nevertheless, their subjective absence must not be adduced as evidence of the presence of disease of the right heart, it being for the most part found that murmurs in the left heart overpower and mask the sounds of the right heart.

Practically, in the absence of all other indications, we have only, as a general rule, the murmurs arising in the left heart to deal with, viz., diastolic and systolic regurgitant murmurs of the mitral valve, and systolic and diastolic regurgitant murmurs of the aortic semilunar valves, and to these may be added murmurs induced by roughnesses and clots in the endocardium, or those resulting from a diseased state of the blood itself.

Nevertheless, it is always necessary to bear in mind that the right heart may also be diseased; and, therefore, when investigating the condition of the heart, it is incumbent not to neglect, if possible, the due ascertaining of this.

Premising that the immediate object now is the discrimination of one cardiac murmur from another, independently of all external sources of confusion, and assuming, in the absence of other signs, that the left heart is the special seat of murmurs, it then becomes necessary, when the existence of a murmur is discovered, to ascertain what is its precise origin in this heart, and whether it be a murmur of flow, or of regurgitation.

Cases frequently present themselves in which there may be but little difficulty in at once referring a sys- tolic murmur, either to a regurgitation through the mitral valve, or to obstruction to the flow of blood through the aortic semilunar valves, or in deciding that disease in these latter valves is the cause both of this and of a regurgitant murmur.

Nevertheless, difficulties in diagnosing each of these murmurs may occur. For instance, a soft, slightly prolonged unurmur at the apex of the heart, synchronous with the diastole and followed by a normal first sound, might reasonably be assumed to be dependent on some obstruction to the even flow through the mitral valve, but without any of those conditions which permit a regurgitation through this valve. It is, however, more than probable, looking to the results of experience, that such a murmur is not due to this cause, but to a regurgitant murmur in the aortic valves, which, being produced by the diastole, is not only synchronous with it, but is found to descend also towards the left apex. Though the possible case of a mitral flow murmur, thus exclusively occurring, is, by way of illustration, here mentioned, I am not aware that I have ever satisfactorily heard it. Doubtless its occurrence as such is very rare; and that it should be so is not surprising, when it is borne in mind that in all probability it would depend on some source of obstruction to a free flow of blood through this valve, which is yet so perfect as a valve as not to permit any regurgitation. Supposing, however, such a condition to exist, its due appreciation may not only be interfered with by the phenomena attendant on the mitral regurgitant murmur, but also by the presence of a regurgitant tricuspid murmur.

The obstructive mitral murmur is, for the most part, however, associated with a systolic regurgitant murmur, and this is usually so much more marked and prominent than the diastolic murmur as to absorb and mask it, and thus become in great measure the only sound heard. This, at least has been the result of my own experience, but Skoda defines the two murmurs as distinctly to be heard; the diastolic murmur immediately preceding the systolic. Under certain circumstances, this clearness and definition of the two murmurs has, certainly, appeared to me not to be altogether unappreciable, especially when a rapid and spasmodic action of the heart induces a quick flow of blood through the valve; but under ordinary circumstances the evenness of the flow and the amount of the mitral valvular obstruction are inadequate to produce a murmur sufficiently loud or marked to be heard as a murmur, separate and distinct from the systolic regurgitant murmur of the same valve.

This latter, the systolic mitral murmur, is, on the other hand, usually defined and to be heard distinctly at the left apex, offering, for the most part, as its chief characteristic, a gentle blowing sound; or this, with only a slight roughness; and, if it be followed by a distinct valvular second sound, presents but little difficulty in the way of satisfactorily recognising its origin. When, however, the valvular second sound is indistinct, it has always appeared to me by no means easy to refer, with precision, the murmur to the mitral valve; for there may, and not infrequently do, exist other causes for a murmur, very similar in sound, and to be heard at the same site. Dr. Walshe (p. 370) specifies particularly that produced by irregularities of surface at the base of the ventricle, and thinks that the distinction between them cannot with positiveness be made in the present state of knowledge; the same perhaps may be said when a murmur is due to the presence of clots in the ventricle.

Fortunately, neither of these causes of confusion are of frequent occurrence. 
The chief practical difficulties, preventing an easy recognition of this mitral systolic murmur, arise from the coexistence of other valvular lesions, inducing a general confusion of sounds, but more especially the presence of a loud systolic murmur in the semilunar aortic valves. This, from being synchronous with the mitral regurgitant marmur, is very apt to mask it, or so overpower it as to prevent its easy recognition.

The basic murmurs, which may be four in number, two systolic and two diastolic, are often very difficult to define; and this is not surprising, considering that their origin is within a very narrow compass, and that the two systolic murmurs are synchronous with each other, as are also the two diastolic, and that these two latter so immediately follow the former, as to be often undistinguishable as any other than a continuous murmur.

Experience, however, has taught us that these basic murmurs have their origin, comparatively, but rarely in the valves of the pulmonary artery, and that, when they do there exist, murmurs will also be found to exist in the aortic valves: therefore, as a question of diagnosis, when a systolic basic murmur alone is heard, there is rarely much difficulty in deciding that its seat is in the aortic valves, and that here, by some sources of obstruction, there occurs interruption to the free and easy flow of the blood. Nevertheless, if this murmur be soft and low in tone, and there be, synchronously with it, a loud systolic regurgitant murmur, which, it may be assumed, has its origin in some deficiency in the mitral valve, the recognition of the aortic murmur may be attended with very considerable difficulty. The presence, also, of an aortic regurgitant murmur, may likewise so mask and overpower a constrictive aortic murmur, as to render it not always easy to decide on the presence of this latter. For the most part, however, if the systolic aortic constrictive murmur be loud and well pronounced, its presence can usually be satisfactorily recognised.

The aortic diastolic or regurgitant inurmur has been shewn above to be sometimes mistaken for the mitral constrictive; on the other hand, this latter, when it is a prominent murmur, and, to a less extent, the same may be said of the constrictive murmur of the tricuspid valve, may mask or prevent a full and due appreciation of the aortic regurgitant. These are not, however, frequent sources of difficulty ; moreover, the murmurs induced in the aortic valves are usually, but not always, sharper and harsher in tone than those of the mitral and tricuspid valves.

With regard to the murmurs emanating from the semilunar valves of the pulmonary artery, and from the tricuspid valves, little is to be advanced here; occasionally a well defined and isolated murmur in the former is to be heard; but these are peculiar cases. For the most part, independently of the comparative rarity of disease in the right heart, murmurs here are so seldom isolated, that, if not obscured by corresponding murmurs in the left heart, they are at any rate so synchronously mixed up with them as to be scarcely distinguishable. The difficulty of diagnosing these murmurs, separately from those of the left heart, solely from the position of the murmur or the character of its sound, has hence appeared to me insuperable, and I cannot but endorse the opinion generally entertained in this respect.

The synchronism which makes of the two sets of valvular sounds but two sounds only, acts more energetically in the case of the murmurs from the prolongation of the sound on the one hand, and the much louder and sharper character of the murmurs emanating from the left heart on the other. In order to arrive at conclusions about the state of the valves of the right heart, it is necessary to call to our aid a full consideration of the attendant symptoms. It will be our part, subsequently, to refer more particularly to these; at present, the sounds emanating from the heart are taken account of alone.

Having, as nearly as one can, settled the precise seat whence proceeds the murmur, there then arises the question as to its nature; whether it be due to organic, or only to functional disturbance, or to an altered condition of the blood, or to the presence of clots in the heart itself. The accurate discrimination of these is really a point of great practical importance.

As a general rule, and perhaps with the sole exception of the mitral regurgitant murmur heard in chorea, and when there are clots in the heart, one may exclude from the category of functional mur. murs all murmurs heard at the apex, regurgitant murmurs at the base, and all murmurs when there is collateral evidence of a diseased heart. There, therefore, only remains, with the above exceptions, the systolic basic murmur with which functional murmurs can be confounded; and, as theiseat of these murmurs is more frequently than otherwise in the pulmonary artery, with the aortic valvular sound distinctly audible, their recognition as functional murmurs is not always unsatisfactory.

For the most part they are hæmic murmurs, and discoverable in those persons whose condition of blood is poor, in cases of incipient phthisis, after excessive hæmorrhages, or when there is menstrual irregularity, and also occasionally after a sustained and increased action of the heart from over-exertion, or from extreme nervous excitement.

The systolic basic murmur heard occasionally in fevers, not typhoid, but rather of the relapsing type, is probably of the same nature and origin. Dr. Stokes, who first called attention to it, says (p. 426) : "This murmur, whatever may have been its seat and actual cause, is clearly to be placed in the category of inorganic murmurs, and its frequent development in the relapse, in cases too where enlargement of the spleen was observed, while at the same time the signs of softening of the ventricle were wanting, makes a strong case in favour of its being in some way connected with a depraved state of the blood."

While speaking of the difficulties that surround the recognition of murmurs in the heart, allusion must not be here omitted to those cases in which, though there may be the elements of murmur, as far as the existence of diseased valves is concerned, yet no murmur exists, or, at any rate, can be recognised. As these cases will be particularly referred to subsequently, it will be here sufficient merely to state that this absence of murmur is chiefly met with where there is, at the same time, evidence of a want of force in the current of blood that passes through them-the main, or rather the absolutely necessary, element towards the production of sound or murmurs. Hence this absence of murmur is very frequently met with when the powers of life are failing, when murmurs are lost in sounds which are inappreciable as belonging either to the category of normal sounds or of murmurs, or to those cases in which the current of blood is impeded by an over-distension of the cavities.

Having now passed in review the causes of the sounds and murmurs proper to the heart, as also some of the difficulties that may prevent their ready and perfect recognition, we will briefly refer to some of the more certain conclusions which may be deduced from the observation of these phenomena.

First, as regards the valvular sounds. If these be all clear and distinct, and free from any murmur, it may be inferred there is the necessary proportion be- 
tween them and the current of the blood; that, in fact, the passage for the blood is not relatively interfered with; so that, looking, on the one hand, to the amount and force of the blood, and, on the other, to valvular structure, it may be inferred as regards the flow of blood, that the valves are in a passive state, and exert no force whereby its flow is resisted or impeded; and, as a correlative of the above, it may be inferred, when valvular murmurs take the place of the normal valvular sounds, that a disproportion between the current of the blood and the opening it has to pass through, does exist, and that the valveopening, whatever may be its condition, acts more or less by interfering with the flow of the current; and this may be due either to excess of force in the current of the blood itself, or to disorder in the valves.

If valve-sounds exist, preceded by a murmur, it may be inferred that the closure of the valve is complete, but that the passage of the blood preceding this closure is interfered with, from one or other of the above causes, so that it has not room to pass through its channels without an eddy being produced. It may also be inferred, if a valve be sufficiently diseased to produce a regurgitant murmur, that the normal valvular sound from this valve will not be heard, the murmur entirely occupying the place of it: the murmur being due to the return of blood through the limited and diseased opening, while the absence of the normal valvular sound is due either to this latter sound being masked by the murmur or to its entire absence, by reason that the diseased condition of the valve does not afford the necessary check to the onward stream of blood. Nevertheless, a valvular sound may occasionally be heard synchronously with the regurgitant murmur, but then it emanates from the corresponding valve in
the other heart.

If the diseased obstruction be in the semilunar valves, the murmur thus resulting will be found to be synchronous with the normal sound of the mitral valve, but, when loud and prolonged, may, to a certain extent, so mask it as to prevent its easy recognition; hence, as a general rule, it may be assumed that a murmur which supersedes both sounds may possibly have its origin in disease of the semilunar valve only, while a murmur, followed by a distinct normal second sound, indicates alteration in the mitral valve. If, however, the mitral murmur be a regurgitant one, it may seriously interfere with the easy appreciation of the second sound; generally, however, it can be detected either in the left, or in the right, heart, and in such case a correct diagnosis can be arrived at.

If the semilunar valves be so diseased as not only to obstruct the systolic flow, but likewise to permit regurgitation, there will either be a very prolonged, or a double, murmur. Experience has shewn this to hold good if the necessary conditions exist both in the aortic semilunar valves, and in those of the pulmonary artery; but, as these latter are rarely the seat of independent lesion, the murmurs generated in them are synchronous with those generated in the aortic valves, and not distinguishable from them.

Independently of all other considerations, the presence of a double murmur indicates disease of the semilunar valves, a double murmur being rarely produced by the mitral valves; for, though these be so contracted as to interfere with a free flow, the slower way in which the blood passes through their valvular opening does not necessarily produce a murmur. Under such circumstances, and they are of frequent occurrence, a regurgitant murmur only is heard, emanating from the mitral valve, while the second murmur is the result of regurgitation through the semilunar valves. Hence, as a general rule, it follows that, if there be a double murmar, the semilunar valves are certainly diseased, and, may be, the mitral also. In order to decide this, it becomes necessary to ascertain the presence or the absence of the first sound. If it be present, there no longer remains any difficulty in the diagnosis.

The explanation of the above may be briefly summed up. In those cases in which the valve, by offering obstruction to the flow of so much blood as is presented to it, induces an eddy, and consequently a murmur, yet being perfect as a valve, the murmur is succeeded by a true normal valvular sound. Where there is permanent patency of the valve, but no obstruction to the flow, there is no normal sound, but there is a murmur. There is also no normal valvular sound where there is both obstruction to flow and imperfect valvular closure, but there is a murmur accompanying both the flow of blood and its abnormal regurgitation; this murmur may be one prolonged murmur or a double murmur; for the most part the former.

The quality and the character of the sound of endocardial murmurs vary greatly in intensity; but little, practically, is, however, to be concluded from this; slight and unimportant disease may be accompanied by the louder sounds, and the extremest of disease by little or even no sound at all. Physically, (and it is this view only that now occupies us) this may depend on various causes, the chief of which are the relative frequency and force of the circulation, on the one hand, and, on the other, the vibrating power of the parts concerned. As are the force, the amount of fluid, and the obstruction, and the vibrating power of the structures concerned, so is the intensity and the quality of the murmur.

The cause of the sound and murmurs having now been, as it is hoped, sufficiently explained, as also their uses in diagnosis, it remains to be seen what may be their value in the prognosis of disease; and here we arrive at the chief object of these papers, which is to determine what practical bearing the physical examination of the heart affords us towards determining not only the nature of its diseases, but their fatal tendencies, or their probabilities of cure or alleviation. In doing this it is not purposed to set forth a full and perfect treatise on them, or to refer to and discuss the recondite observations of others, but rather to state the results and conclusions of a personal experience. In doing this, I feel the responsibility of the task, and the certainty of the incompleteness of its fulfilment. The grave difficulty of the imperfectness both of accurate observation, and of its correct rendering, immediately and prominently presents itself. Doubtless the sounds and physical indications, which, in modern phraseology, may be termed the objective qualities, are defined and true to themselves; not so the subjective qualities of the observer, for these vary not only in different individuals, but also in each individual at different periods of his professional life. The sounds emanating from the heart may be simple, and must be defined and truthful; but the ear that listens to them may be imperfect. Supposing, however, this difficulty to be surmounted, and that the sounds emitted are equally recognised by all, there remains the further difficulty that thelesson tobe learned from them is limited and inconclusive, and then we yet further learn that correct diagnosis can only be arrived at by a consideration of other elements. Nevertheless, their study is doubtless useful to this end.

But if this be their limited use in diagnosis, in prognosis it becomes still more limited. Here the unaided sounds and murmurs emitted by the heart are
anything but conclusive, there being many diseases 
of this organ of a most fatal tendency, in which they are absent, while some, in which they loudly present themselves, are not so; hence, in what follows, it will be seen that other actions, vital and mechanical, must necessarily be duly considered, so that, while pointing out the diagnostic value of the sounds and murmurs, as regards disease and its consequences, it will also be necessary to show that in themselves and without the aid of other concomitant symptoms, they cannot be relied on as the sure and certain indicators of the precise condition of diseased structure, or of its relative gravity and fatality.

[To be continued.]

\section{ANEURISM OF THE BRACHIAL ARTERY CUREI) BY MANIPULATION.}

\author{
By F. POOLE LANSDOWN, Esq., \\ Surgeon to the Bristol General Hospital.
}

J. D., aged 45, an insurance agent, living at Newport, came under my care at the Bristol General Hospital on the 30th of January, 1866. He was a tall spare man, with only one leg, his left thigh having been amputated twenty-four years previously for inflammation of the knee-joint. It was removed rather high up, so that he had used a crutch ever since. He first noticed the swelling in his left arm about a month ago. When getting out of bed one morning, his attention was called to it by the pulsation; it gave him no pain. He at once consulted a surgeon, who applied a bandage over it, and advised him to go up to Bristol.

The tumour was fusiform, of about the size of a duck's egg, soft and pulsating, expanding in all directions; it was situated about two inches below the posterior fold of the axilla, in the course of the brachial artery. On the current of blood being stopped, it was easily emptied, gradually refilling when the pressure was removed. A loud bruit was to be heard over the tumour.

As he had to return home on business, he did not come into the house until February 2nd, when, on examining the aneurism, I was at once struck with its hardness and want of pulsation. On questioning the patient, he told me that he noticed the change in the swelling on the day after his visit to the hospital. The pulse at the wrist could just be felt, though very feeble. There was not the least pulsation in the tumour, nor was any bruit to be heard. The left hand was a little cooler than the right. I ordered him to remain in the borizontal position, on a simple diet.

Feb. 5th. The tumour was sensibly smaller; and the radial artery was more easily felt at the wrist.

Feb. 7th. The circumference of the arm over the tumour was less by half an inch than on the 5th. The radial pulsation was stronger. A branch of the superior profunda along the outer side of the arm, apparently nearly as large as the radial artery, was seen pulsating.

Feb. 19th. The circumference of the arm was one inch less than when first measured, showing how much the tumour had subsided. He was made an out-patient to-day.

July 31st. The patient presented himself to-day, quite well, and free from any inconvenience. A fibrous cord was all that remained of the aneurism.

Remarks. It appears to me that the above is an interesting case, both from the rarity of its situation and the rapidity with which the aneurism was cured. The constant use of the crutch was the apparent cause of the disease; and I attribute the cure to the

detachment of a portion of clot during the examination at our first interview. I handled it rather more freely than I should otherwise have done, in order to show the students the diagnostic signs of aneurism.

\section{aneports}

or

\section{HOSPITAL PRACTICE: METROPOLITAN AND PROVINOIAL.}

MIDDLESEX HOSPITAL.

Clinical Remarks on Fever.

I.-CASE OF TYPHUS FEVER, TERMINATING IN DEATH FROM CARDITIS IN THE FOURTH WEEK OF THE DISEASE: AUTOPSY.

(Under the care of Dr. Murchison.)

The subject of this case was a middle-aged woman, who had for some time been lying in one of the surgical wards at the top of the house, on account of a tumour in the pelvis. As she was far away from the medical wards, in which there had lately been two or three cases of typhus, it could nct at first be made out how she had caught the infection; but, on investigation, it was ascertained that one of the nurses who attended to the fever cases was in the habit of visiting a patient occupying the bed next to this woman. This was the only mode of propagation of the fever-poison that could be made out. This circumstance, however, did not come to light till afterwards ; and for the first few days the diagnosis was regarded as doubtful between continued fever and some cerebral affection; for, during the first week, very severe headache was complained of, and there was distressing vomiting, the patient bringing up everything that she took, even water. At the end of the first week, albumen was detected in the urine; and, on auscultation, a double friction-sound, clearly pericardial, was heard over the base of the heart. The appearance of the characteristic typhusrash on the eighth day, which was unusually late, settled all doubt as to the nature of the case. The eruption consisted of red blotches, which gradually became brown, and then petechial in the centre. About the end of the secondweek, signs of amendment began to show themselves; the pulse came down to 124, and kept on falling; the temperature also fell; and for five or six days the patient went on improving; her appetite returned, and she could eat even meat. On the twenty-first day of her illness, however, she was not so well again; she was restless, fidgety; the pulse and temperature had gone up again, the latter being even higher than it had been before any amendment took place.

There never being any relapse in typhus, as there sometimes is in typhoid, Dr. Murchison suspected that the sudden aggravation of the symptoms was due to some visceral complication, probably pulmonary congestion or inflammation, which are generally apt to be set up in such cases. On careful examination of the lungs, however, nothing could be detected in them which could account for the patient's dyspncea, the rise in the temperature, and the increase in the number of pulsations at the wrist. But, when the heart came to be examined, the source of the mischief appeared to be made out. A systolic endocardial murmur was heard 